\title{
Effects of Fermented Jackfruit Leaf and Pulp Beverages on Gut Microbiota and Faecal Short Chain Fatty Acids Content in Sprague-Dawley Rats
}

\author{
Yun-Shin Sew ${ }^{1}$, Sarah Sabidi ${ }^{2}$, Shazwan Abdul Shukor ${ }^{1}$ and Soo Peng Koh ${ }^{2 *}$ \\ ${ }^{1}$ Biotechnology and Nanotechnology Research Centre, Malaysian Agricultural Research Development Institute, Malaysia \\ ${ }^{2}$ Food Science and Technology Research Centre, Malaysian Agricultural Research Development Institute, Malaysia
}

*Corresponding author: Soo Peng Koh, Food Science \& Technology Research Centre, Malaysian Agricultural Research and

Development Institute, Malaysia

\section{ARTICLE INFO}

Received: 幽 August 03, 2020

Published: 慧 August 13, 2020

Citation: Yun-Shin Sew, Sarah Sabidi, Shazwan Abdul Shukor, Soo Peng Koh. Effects of Fermented Jackfruit Leaf and Pulp Beverages on Gut Microbiota and Faecal Short Chain Fatty Acids Content in Sprague-Dawley Rats. Biomed J Sci \& Tech Res 29(3)-2020. BJSTR. MS.ID.004817.

Keywords: Fermented Jackfruit; Beneficial Microbes; rRNA Sequencing; Functional Beverage
ABSTRACT

Consumption of fermented products including those prepared using Symbiotic Culture Of Bacteria And Yeast (SCOBY) has been well associated with their health promoting effects. In this study, we investigated the changes of gut microbial community structure and faecal SCFAs content in Sprague-Dawley rats following SCOBY Fermented Jackfruit Leaf (FJL) and Fermented Jackfruit Pulp (FJP) diet interventions. The study was conducted by force-feeding adult rats with individual FJL and FJP diet, while normal water diet was given to the control for 4 weeks prior to faecal sample collection for 16S rRNA sequencing and SCFAs analyses. Operational Taxonomy Unit (OTU) analysis showed that a higher gut microbiota diversity in both fermented diet treated groups compared to control. The consumption of fermented jackfruit diets resulted in gut microbiota with enriched beneficial microbes and lower Firmicutes: Bacteriodetes ratios. More specifically, the abundances of methane-producing microbe (Methanobrevibacter) and butyric acidproducing microbes (Faecalibacterium, Coprococcus and Clostridium) increased markedly in FJL and FJP treated group, respectively, suggesting that these diets plausibly modulate host energy expenditure. Overall, it was evident that SCOBY FJL and FJP are emerging functional beverages due to their associated health benefiting properties.

Abbreviations: SCOBY: Culture of Bacteria and Yeast; FJL: Fermented Jackfruit Leaf; FJP: Fermented Jackfruit Pulp; OUT: Operational Taxonomy Unit; CFFC: Collection of Functional Foods Cultures

\section{Introduction}

The jackfruit (Artocarpus heterophyllus L.) is one of the Southeast Asia's popular tropical fruit trees, of which the extracts from its leaf and pulp contain valuable phytochemicals such as phenolics, flavonoids, phytosterol, carboxylic acids, coumarins and saponins $[1,2]$. Due to its multiple medicinal properties and health benefits [3-5], there is a great potential for this fruit to be applied in the food processing industry. For instance, it offers good substrate for food fermentation process that provides value adding effects to the functional properties of jackfruit. Fermentation technology has been long known to improve the sensory quality of the food such as taste and aroma, refined texture, as well as extended shelf-life [6]. During fermentation, the food complexes are broken down by microbes and new bioactive compounds are produced which could improve the overall nutritional and functional contents of fermented food. Kombucha fermented tea broth which is prepared using mixed culture of bacteria and yeast has been reported to have antimicrobial and antioxidant activities [7,8] and other health promoting effects $[9,10]$. Likewise, the fermented jackfruit leaf and pulp beverages prepared using selected symbiotic culture of bacteria and yeast (SCOBY) strains from MARDI's Collection of Functional Foods Cultures (CFFC) were reported to exhibit high antioxidant activities [11]. 
Studies have shown that the microbial composition in different parts of gut system has great implications to biological and physiological aspects of the host, which include host nutrient metabolism, strengthening mucosal barrier, gut protection, maintenance of gut immunity and regulation of xenobiotic and drug metabolism [12]. Factors that structuring gut microbiota are physiological and environmental dependent, primarily contributed by host genotype, habitat, dietary intake and antibiotics use [13]. Certain type of fermented products particularly for those supplemented with commercial probiotic strains could facilitate a significant amount of beneficial microbes to be delivered to the gut system and thereby improve the functional microbial composition [14]. Moreover, the resulting bioactive end products from fermentation derived microbes could interact with host microbiota and impacted positively on gastrointestinal health and diseases [15], rheumatic disease [16], immune system [17], brain and cognitive health of human beings $[14,18]$.

The gut microbiota in the colon are capable of degrading the dietary fibers, with majority of these microorganisms are saccharolytic which use carbohydrates as fermentation substrates, giving rise of Short Chain Fatty Acids (SCFAs) and gases [19] as the end products. Major SCFAs such as acetic acid, butyric acid, and propionic acid make up about $90-95 \%$ of total SCFAs content which present in the colon of rat and human [20]. These compounds are formed as the by-products of gut microbes during fermentation of non-digestible polysaccharides and can be used as a fuel source for the host $[21,22]$. Changes in SCFA composition following consumption of fermentable carbohydrates were shown to be well associated with improved health conditions in the context of glucose homeostasis, blood lipid profile, reduction of body weight and colon cancer risk [23-26]. Therefore the faecal SCFAs level could provide crucial information about the composition, metabolism and function of gut microbiota within certain gut environment responding to a particular dietary modulation.

Majority of microbes residing in gut lumen are beneficial to human, however many of those microbes are somewhat uncultivable [27]. With the unprecedented advances in next generation sequencing technology, identifying uncultivable gut microbes have been made possible by using 16S ribosomal RNA (rRNA) sequencing approach. By targeting on the hypervariable regions (V1-V9) contained in bacterial rRNA gene, this approach could distinguish and profile almost all bacterial species residing within a natural community of gut ecosystem [28,29]. In this study, we aimed to investigate the changes in rodent gut microbiota mediated by SCOBY fermented jackfruit leaf and pulp beverage diets through 16S rRNA sequencing. In addition, we profiled and compared the primary SCFAs between individual fermented diet treated and control group. The findings obtained from this study can help us to improve our understanding on the diet-host-microbe interactions related to SCOBY fermented products.

\section{Materials and Methods}

\section{Preparation and Fermentation Process of Jackfruit Ma- terials}

Fruits and leaves of Artocarpus heterophyllus L. were obtained from a local jackfruit farm of Pahang state in Malaysia. The jackfruit fruit pulp and leaves were thoroughly cleaned and air dried before they were ground into powder form to be used as substrates for producing Fermented Jackfruit Pulp (FJP) and leaf (FJL) beverages, respectively. Two types of microorganisms: a) yeast (Dekkera sp.) and b) acetic acid bacteria (Komagataiebacter sp.) were selected from MARDI's Collection of Functional Food Cultures for jackfruit fermentation process. Jackfruit suspension was prepared at the concentration of $5 \%(\mathrm{w} / \mathrm{v})$ and inoculated with the mixture of yeast and acetic acid bacteria at colony count of $1 \times 10^{8} \mathrm{cfu} / \mathrm{ml}$ prior to fermentation process at $30^{\circ} \mathrm{C}$ for 7 days. The supernatant was collected by centrifuging the fermented suspension at 10,000 rpm for $10 \mathrm{~min}$ to separate the microbes and substrate residues and stored at $4^{\circ} \mathrm{C}$ until further use as treatment samples.

\section{Animals Groups and Experimental Design}

Six-week old Sprague-Dawley rats were kept in the cage and acclimatised for 2 weeks with standard conditions at $25 \pm 2^{\circ} \mathrm{C}$ with 12 $\mathrm{h}$ light/dark cycles at MARDI Animal, Toxicology and Reproductive Centre. All rats were supplied with distilled water and commercial laboratory rodent pellets. All experimental procedures were conducted according to the guidelines provided by Animal Ethics Committee of MARDI (MARDI Ethics Approval ID 20170420/R/MAEC 00008). A total of nine female rats were divided into 3 groups, a) fermented jackfruit leaf (FJL); b) Fermented Jackfruit Pulp (FJP) and c) control (CTL). The rats were force-fed with fermented beverage or water using oral gavage technique according to a dosage of $4000 \mathrm{mg} / \mathrm{Kg}$ body weight. All rats were given normal diet throughout the experiment.

\section{Faecal Sample Collection and DNA Extraction}

The faecal samples were collected from rats of each group: a) Fermented Jackfruit Leaf (FJL); b) Fermented Jackfruit Pulp (FJP); c) control (CTL) after 28 days experimental period. The faecal specimens were subjected to DNA extraction using NucleoSpin tissue mini kit (Macherey-Nagel, Germany) according to the manufacturer's instructions. DNA concentration was determined using Nanodrop 1000 spectrophotometer (Thermo Scientific, IL, USA) and the purity was obtained based on the absorbance at A260/A280. The quality of isolated genomic DNA was monitored on $1 \%(\mathrm{w} / \mathrm{v})$ agarose gel. The isolated DNAs were then stored at $-80^{\circ} \mathrm{C}$ until further use.

\section{6s rRNA Libraries Construction and Sequencing}

16S rRNA genes were amplified by specific PCR primers (16S V4:341F/806R) (341F: CCTAYGGGRBGCASCAG and 806R: GGACTACNNGGGTATCTAAT) using Phusion High-Fidelity PCR Master 
Mix (New England Biolabs, Ipswich, MA, USA) and PCR program (4 min at $94^{\circ} \mathrm{C} ; 35$ cycles of $1 \mathrm{~min}$ at $94^{\circ} \mathrm{C}, 1 \mathrm{~min}$ at $50^{\circ} \mathrm{C}$ and $2 \mathrm{~min}$ at $72^{\circ} \mathrm{C}$; and finally $10 \mathrm{~min}$ at $72^{\circ} \mathrm{C}$ ). The resulting PCR products were mixed with equal volume of $1 \mathrm{X}$ loading buffer and subjected to $2 \%$ $(\mathrm{w} / \mathrm{v})$ agarose gel electrophoresis for detection. The DNA fragments between 400-450 bp were fractionated and purified using Qiagen Gel Extraction Kit (Qiagen, Hilden, Germany). Sequencing libraries were constructed using PCR products from each group and TruSeq ${ }^{\circledR}$ DNA PCR-Free Sample Preparation Kit (Illumina, USA) according to the manufacturer's instructions. The quality of library was assessed using the Qubit ${ }^{\circ}$ 2.0 Fluorometer (Thermo Scientific, Waltham, USA) and Agilent Bioanalyzer 2100 system (Agilent Technologies, Palo Alto, CA). Sequencing was performed on Illumina HiSeq 2500 platform (Norcross, GA, USA) with 250 bp paired-end reads generated.

\section{Bioinformatics and Data Analysis}

Paired-end reads were assigned to samples and merged using FLASH v. 1.2.7 [30] to generate raw tags. The raw tags were filtered using QIIME v. 1.7.0 [31] to obtain high-quality clean tags. For chimera detection, the tags were compared with the 'Gold' reference database [32] using UCHIME algorithm before the sequences were removed to obtain the effective tags. Sequence analysis was performed by UPARSE (v. 7.0.1001) [33] where unique sequences with $\geq 97 \%$ similarity were clustered into operational taxonomic units (OTUs). The representative OTUs were annotated based on Green Gene Database [34] and taxonomic assignments provided by RDP classifier (v. 2.2) [35]. Analysis of phylogenetic relationship among different OTUs was performed using PyNAST software (v. 1.2) [36] which enables multiple sequence alignment against the "Core Set" dataset in the GreenGene database. The OTUs were normalized by dividing the abundance of each organism and its predicted $16 \mathrm{~S}$ rRNA gene copy numbers. The unique and shared OTUs among groups were presented in a venn diagram constructed using jvenn Results
[37]. OTU abundance data was used to calculate alpha diversity (Shannon index and Chao 1), observed species richness and rarefaction estimates using Mothur [38]. The compositional changes in the gut microbial community were investigated by estimating the percent OTU abundance of specific bacteria (at the phylum level) in individual sample group. The values of relative abundances of OTUs between individual fermented diets treated and control group were subjected to $\log _{2}$ transformation. The $\log _{2}$ transformed values were then used for Pearson correlation and hierarchical clustering with heat map analysis in Multi experiment Viewer (MeV, v. 4.2) [39].

\section{Analysis of Short Chain Fatty Acids (Scfas) in Rat Faecal Samples}

Approximately $0.3 \mathrm{~g}$ faeces was added into a tube containing lysing matrix type E (MP Biomedicals, USA) and homogenized in $3 \mathrm{ml}$ sterile distilled water by vortexing to get faecal suspension. The suspension was adjusted to a final $\mathrm{pH} 3$ by adding $50 \mu \mathrm{l}$ of 10 $\mathrm{M} \mathrm{HCl}$ and vortex vigorously for $10 \mathrm{~min}$. The homogenate was then centrifuged at $5,000 \mathrm{rpm}$ for $10 \mathrm{~min}$ at $4^{\circ} \mathrm{C}$. The SCFAs containing supernatant was filtered using a $0.22 \mu \mathrm{m}$ syringe filter and the faecal extracts were stored at $-20^{\circ} \mathrm{C}$ until further analysis. The composition of SCFAs was analysed using gas chromatography (Agilent $6890 \mathrm{~N}$, USA) equipped with Flame Ionization Detector (FID). One microlitre faecal extract was injected into gas chromatography machine using auto injector system. The major SCFAs (i.e. acetic acid, propionic acid and n-butyric acid) were identified and quantified based on a standard calibration curve. All samples were analysed in triplicate. The concentration of SCFAs was recorded as mean $\mu$ mole per gram faeces ( $\mu \mathrm{mol}$ g- 1$) \pm$ standard error. The average of percentage change $(\%)$ was calculated from triplicate samples for individual SCFA in control and treated groups. Student's t-test was used to determine significant differences between values at $1 \%$ significance level $(\mathrm{p}<0.01)$ and $5 \%$ significance level $(\mathrm{p}<0.05)$.

\section{S rRNA sequencing analysis}

A

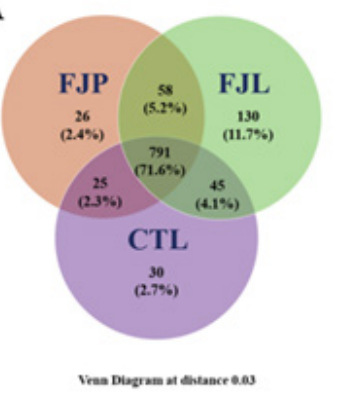

B

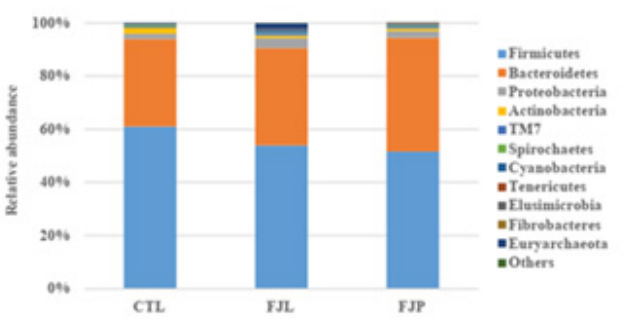

Figure 1: Gut microbiome under diet intervention of Fermented Jackfruit Pulp (FJP), fermented jackfruit leaf (FJL) and normal water (CTL).

(A) Venn diagram describing distribution of unique or shared OTUs across different diet groups, where the observed OTUs were calculated at $3 \%$ distance level.

(B) Relative abundances of gut microbiota (OTU basis at the phylum level) that underwent fermented diet (FJP, FJL) and normal water (CTL) diet. 
To investigate the effects of jackfruit fermented diets on gut microbiota, we compared the changes in faecal microbial composition between treatment and control groups. It was observed that the raw sequences for CTL, FJL and FJP group were 153,329, 155,746 and 145,155 , respectively (Table 1). By assigning sequences with $\geq 97 \%$ similarity to the same OTUs using UPARSE software, there were an average of 105,565, 109,364 and 100,168 total effective tags for CTL, FJL and FJP group, respectively. Species annotation showed that $93-96 \%$ of total effective tags were assigned as taxon tags with OTU number ranging from 891 to 1,024 for all groups analysed (Table 1). These results indicated that OTU numbers were higher in the fermented diet treated groups (FJL and FJP) as compared to control (CTL). The distribution of gut bacterial commu- nities across fermented diet treated groups was next investigated. The identified gut microbiota network structures include: i) unique OTUs associated with the FJL treatment; ii) unique OTUs associated with the FJP treatment; iii) unique OTUs associated with CTL; iv) OTUs shared between groups i and ii; v) OTUs shared by groups $i$ and iii; vi) OTUs shared by groups ii and iii; and vii) OTUs shared by all groups (Figures 1A \& 1B). The total richness calculated at 0.03 dissimilarity cut-off across all groups was 1,105 OTUs. Approximately $71.6 \%$ (791 OTUs) of total bacterial communities were identified as core microbiome across all diets. Interesting to note that FJL showed the highest percentage of unique OTUs (11.7\%) among the samples.

\section{Diversity of the Bacterial Community in Treated and Control Rat Faecal Samples}

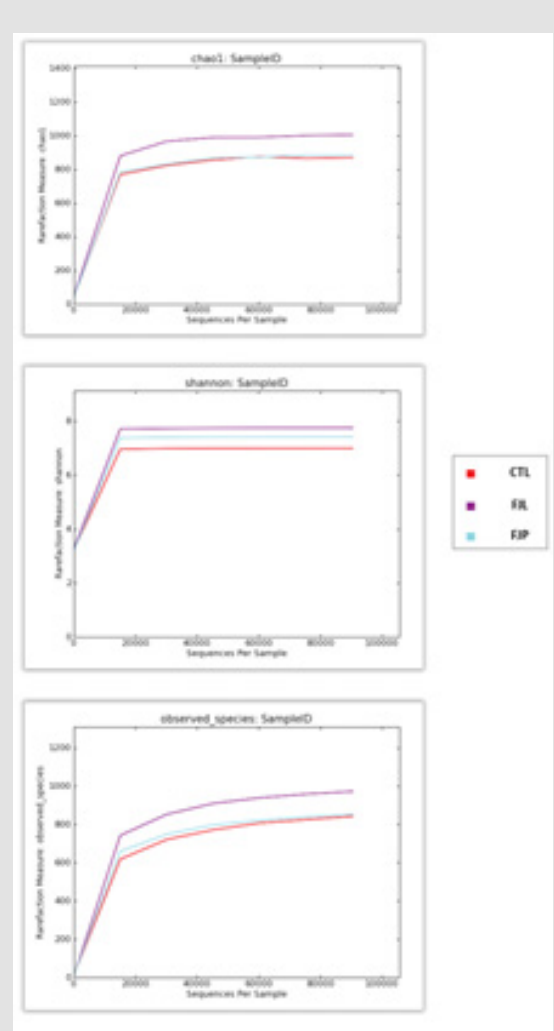

Figure 2: Rarefaction curves of fermented diet (FJP, FJL) treated and control groups according to 3 different algorithms. Chao 1

(A) Shannon and

(B) Observed species

(C) Alpha diversity analyses. Curves were calculated based on OTUs at $97 \%$ similarity.

Alpha diversity analysis was performed for the identification of community richness (Chao index), community diversity (Shannon index) and observed species for assessing the sequencing depth. There were no marked differences in bacterial diversity across all groups with any of the alpha diversity estimators used. Rarefaction curves for bacterial DNA sequences approached a plateau level of approximately 2,000 sequences for each group analysed (Figure 2).

\section{Gut Microbial Changes in Rats Subjected to Different Di-}

\section{ets}

The most abundantly found bacteria at the phylum level for all groups according to OTU analysis are shown in Figure 1B. Among those, Firmicutes and Bacteroidetes were the two bacterial phyla with the highest abundances, consisting of 52-61\%. There were pronounced reductions in Firmicutes: Bacteroidetes ratio after one month of fermented diet consumption, 1.47 for FJL and 1.21 for FJP as compared to 1.86 in the CTL. The effects of fermented diets on gut microbiota composition were further examined by fold change analysis of gut microbial abundance relative to CTL (Figure 3). Basically, the overall relative microbial changes between FJL and FJP treatments showed similar trend. Notably, there were concurrent increases in the relative abundances of Bacteroidetes, Proteobacte- 
ria, TM7, Cyanobacteria and Tenericutes, in constrast to decreases in Firmicutes, Actinobacteria and Spirochaetes for both treatments. Worth mentioning that the abundances of and Cyanobacteria increased at least by 2 -fold in FJL treated group $(\mathrm{p}<0.01)$. However, there were a few bacterial phyla responded inversely to treatments such as Fibrobacteres, Euryarchaeota and Elusimicrobia. Among these, the abundances of Euryarchaeota in particular increased by at least 2.7-fold $(\mathrm{p}<0.01)$ in FJL treated group in contrast to a drastic reduction of 4.4-fold in FJP treated group.

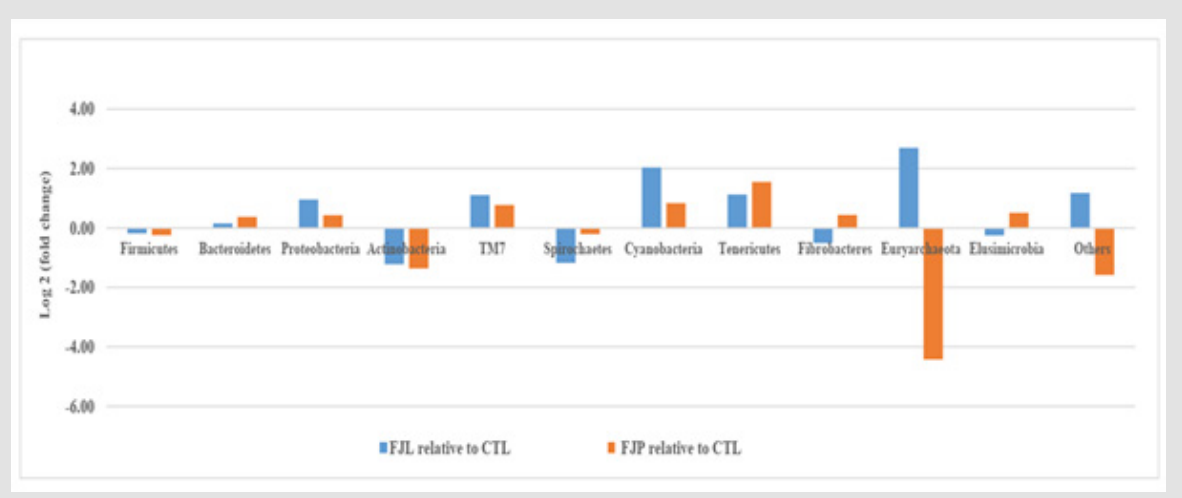

Figure 3: Changes in gut microbiota following fermented diet (FJP, FJL) and normal water (CTL) diet. Values represent log 2 fold changes of top 12 phylum level taxonomy microbial composition of individual fermented diet treated relative to their control.

\section{Hierarchical Clustering Analysis of Relative Abundances of Gut Microbiota}

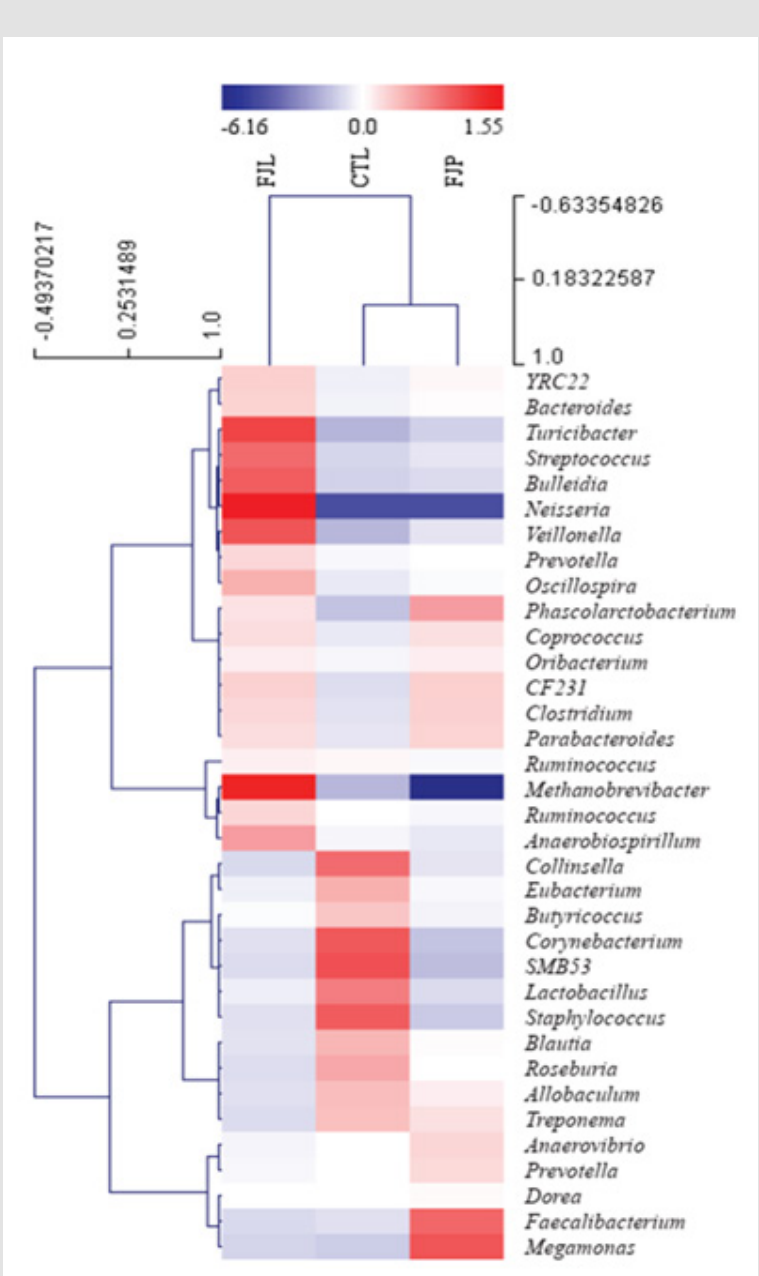

Figure 4: Bacterial community heat map based on the abundance of gut microbiota in the samples. The columns represent individual samples while rows represent OTU abundance value (at the genus level) in log2 scale. The hierarchical clustering was performed using Pearson correlation metric with average linkage method in $\mathrm{MeV}$ software. 
For a more comprehensive comparison of diet associated gut microbial changes, we performed hierarchical clustering analysis of Pearson correlation on the relative abundance of gut microbiota (OTUs basis at the genus level) (Figure 4). Based on the analysis, both fermented diets affected positively to the growth of certain gut microbes which belong to the phylum of Firmicutes (e.g. Phascolarctobacterium, Coprococcus, Oribacterium and Clostridium) and Bacteriodetes (e.g. YRC22, Bacteroides, CF231 and Parabacteroides). Notably, these groups of bacteria showed strong relationships among each other ( $R>0.85)$. Nevertheless, the results show that a cluster of Firmicutes and Bacteriodetes were preferentially enriched their abundances responding to individual fermented diet. For instance, the growth of bacteria at genus level of Firmicutes namely Turicibacter, Streptococcus, Bulleidia, Neisseria, Veillonella, Oscillospira and Ruminococcus were particularly enhanced in the FJL treated group, in comparison to Faecalibacterium and Megamonas that abundantly present in FJP treated group. In addition, a drastic increment of Methanobrevibacter was noticed for FJL treatment. It is worth noting that some opportunistic microbes e.g. Corynebacterium, Staphylococcus, SMB53, Anaerovibrio and Eu- bacterium were consistently decreased in their abundances across diet treatments.

\section{Analysis of Short Chain Fatty Acids Content in The Rat Faecal Samples}

To investigate the changes of SCFAs in the gut system corresponding to individual fermented jackfruit diet, the content of major SCFAs such as acetic acid, propionic acid and butyric acid in the faecal samples of all groups were determined. Percent changes of SCFAs content between pre- and post-intervention were calculated for each group (Figure 5). The results showed that major SCFAs increased notwithstanding a remarkably reduction of $50 \%$ butyric acid in the control group during the diet intervention. Acetic acid content decreased by $25.8 \%$ and increased by $35.7 \%$ in FJL treated and FJP treated groups, respectively. Propionic acid were consistently reduced by approximately $25 \%$ under both diet treatments. Butyric acid content were found reduced by $13.3 \%$ for FJL treated group but slightly increased for FJP treated group, and both treatment groups differed significantly from control diet group $(\mathrm{p}<0.05)$.

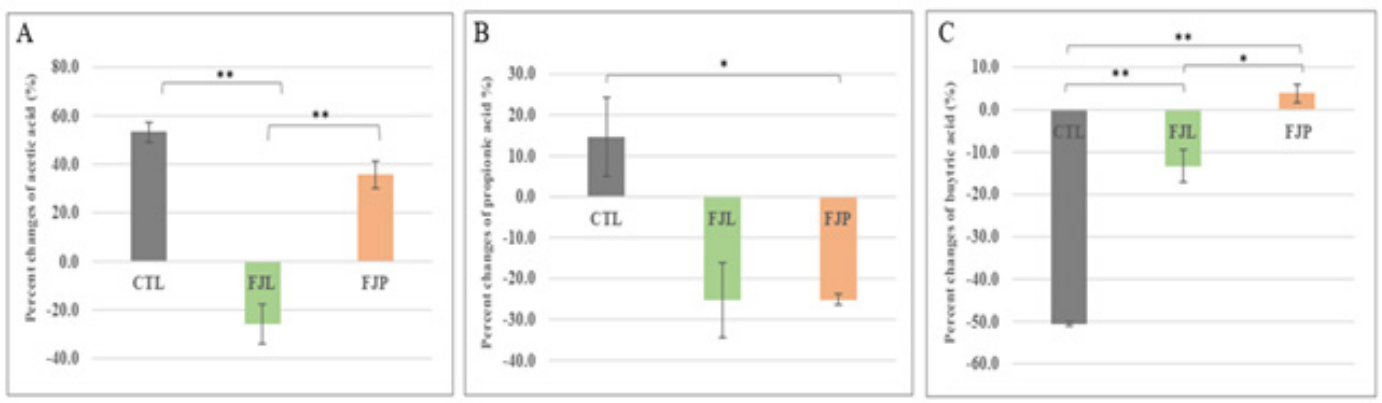

Figure 5: Changes of SCFAs content in the faecal sample of fermented diet (FJP, FJL) and normal water (CTL) treated group.

(A) Values represent the mean percent change of acetic acid,

(B) Propionic acid and

(C) Butyric acid between pre-and post-intervention for respective treatment and control group. Significant differences between groups by Student's t-test analysis are marked with asterisk(s) for significance level at $\mathrm{p}<0.05\left(^{*}\right)$ and $\mathrm{p}<0.01\left({ }^{* *}\right)$, respectively.

\section{Discussion}

Studies associated with fermented food and beverages specific microbiota so far have been focusing on fermented dairy food and beverages such as cheese, yogurt, fermented milk and kefir $[40,41]$. While microbiome studies related to non-dairy fermented fruits and vegetables, soy and cereal products are relatively lacking. Both dairy and non-dairy based fermented food play important roles in delivering probiotics, which give significant impacts to human health. In fact, fermentation process with different combination of food microbes and substrate resulted in different health benefiting properties, representing the uniqueness of a particular fermented product. This work demonstrates how were microbial communities structured and SCFAs changed in the gut system following a short term consumption of fermented jackfruit leaf and pulp beverages in rat model system. Our sequencing results showed that the faecal microbial compositions at the OTU number basis under FJL treatment were $15 \%$ more in comparison to control. Whereas, FJP treated group showed comparable OTUs with the control. Similarly, Venn diagram analysis showed higher number of unique OTUs were assigned to FJL treated group, with 5 times greater than FJP treated group. These results suggest that between fermented diets, FJL provided a higher number of unique phylotypes and a likelihood to mediate a higher microbial diversity in gut system.

Based on taxonomic distribution of gut microbe OTUs it was found that all groups were dominated by Firmicutes, Bacteroidetes and Proteobacteria. These are the major players in the gut microbiota as reported in other rodent model studies $[42,43]$. The ratio of Firmicutes to Bacteroidetes ( $\mathrm{F} / \mathrm{B}$ ratio) has been suggested as an 
indicator for host metabolic health $[44,45]$, and the average ratio of $\mathrm{F} / \mathrm{B}$ in healthy lean rats was approximately 1.48 in contrast to 4.65 in obese rats [46]. In this study, both fermented diets resulted in a remarkable reduction in F/B ratio, 1.47 or lesser compared to control, implying a better health effect upon the consumption of both fermented diets. However, FJP was found to demonstrate a lower F/B ratio than FJL, indicating that Bacteroidetes and Firmicutes abundances were altered to a greater extent by FJP treatment. Both fermented diets were found to exert similar effects on Bacteriodetes, as the abundances of Bacteroides, Parabacteroides and YRC22 were collectively increased compared to control. In contrast, individual fermented diet preferentially affected the growth of certain Firmicutes. The abundances of Veillonella, Streptococcus, Oribacterium and Megamonas were markedly increased under FJL, whereas Ruminococcus, Coprococcus, Clostridium, Bulleidia and Faecalibacterium increased substantially under FJP treatment. These Firmicutes composition differences (which is the largest group of gut microbiota structure) between diets are potentially representing gut microbial signatures associated with individual fermented jackfruit diet.

Among the top 12 predominant phyla, the relative abundance of Euryarchaeota was remarkably altered following diet intervention. FJL was found to promote the growth of Euryarchaeota by 2.7 -fold compared to the normal diet. Conversely, FJP retarded Euryarchaeota abundance as much as 4.4-fold. These data coincided with the abundance levels of Methanobrevibacter (the genus of Euryarchaeota) in the respective treated groups as shown in hierarchical clustering analysis. Species within Methanobrevibacter are aceticlastic methanogens due to their capability of utilizing the methyl group of acetic acid as substrate during the conversion of acetic acid to methane and carbon dioxide [47]. These are best explained with a significant reduction of faeces acetic acid content in FJL treated in contrast to remarkable increase in FJP treated group as revealed by SCFA analysis. Reduction of Methanobrevibacter was found to be a significant factor leading to obesity in human [48]. More recently, a high abundance of Methanobrevibacter was shown to be beneficial for low calorie food intake or malnourished individuals as it would lead to an increase of energy efficiency [49].

It is noteworthy that both FJL and FJP diets positively affected the abundance levels of butyric acid-producing bacteria such as Coprococcus and Clostridium, while the abundance of Faecalibacterium was particularly enhanced under FJP diet. These findings are in accordance with significant increases of faecal butyric acid content observed in treated groups after diet intervention. According to Hippe, Zwielehner [50], the gene copy number of butyryl-CoA:acetate CoA-transferase which is responsible for butyric acid production significantly reduced as ageing progresses. This finding was in agreement with what we observed in our control group. Both fermented diets especially for FJP resulted in a significant increase in butyric acid content, implying a health benefiting effect in ameliorating the loss of butyric acid in aged rats. Butyric acid plays an important role in maintaining a healthy colon [51], important for brain health [52] and cardiovascular function [53] as well as inhibiting the growth of colorectal and breast cancer cells $[54,55]$. Butyric acid compound was also shown to be the preferred energy substrate for colonocytes [56,57], involved in mechanism of up-regulating the mitochondria function and thereby promoting energy expenditure [58]. Clearly, both fermented jackfruit beverages affected gut microbiota and colonic SCFAs positively, have great implications on host energy metabolism and various beneficial effects on mammalian nutrition.

\section{Conclusion}

Taken together, this study provides insights into changes in the gut bacterial communities mediated by fermented jackfruit leaf and pulp diets, resulted from an interplay between diets, host and gut microbiota. Both fermented diets impacted positively and diversified gut microbial composition, presenting unique microbial signatures with prospective functionality. More interestingly, both fermented diets could modulate the host energy expenditure by promoting higher abundance level of energy related microorganisms such as methane- and butyric acid-producing microbes in FJL and FJP, respectively. The present study also exemplifies fermented jackfruit products prepared using SCOBY strains as a promising functional fermented product with great potential, an alternative to traditionally or commercially made fermented products prepared using probiotics strains such as lactic acid bacteria and Bifidobacteria. Further works on the identification of bioactive ingredients from fermented jackfruit leaf and pulp beverages are currently underway which could help to elucidate the mechanism of action related to health benefiting effects of these fermented products.

\section{Funding}

This work was conducted with the support from Horticulture Research Centre, Malaysian Agricultural Research and Development Institute and funded by Malaysia Development Fund RMK 11 (P21003004050001).

\section{Conflicts of Interest}

The authors declare no conflict of interest.

\section{Ethical Approval}

The animal experiments were approved by Animal Ethics Committee of MARDI (20170420/R/MAEC00008) and all experimental procedures were carried out in accordance with the approved guidelines.

\section{References}

1. Jagtap U, Bapat V (2010) Artocarpus: A review of its traditional uses, phytochemistry and pharmacology. J Ethnopharmacol 129(2): 142-166.

2. Singh A, Maurya S, Singh M, Singh U (2015) Studies on the phenolic acid contents in different parts of raw and ripe jackfruit and their importance in human health. Int J Appl Sci 2(2): 69-73.

3. Khan M, Omoloso A, Kihara M (2003) Antibacterial activity of Artocarpus heterophyllus. Fitoterapia 74(5): 501-505. 
4. Omar HS, El Beshbishy HA, Moussa Z, Taha KF, Singab ANB (2011) Antioxidant activity of Artocarpus heterophyllus Lam. (Jack Fruit) leaf extracts: remarkable attenuations of hyperglycemia and hyperlipidemia in streptozotocin-diabetic rats. The Scientific World Journal 11: 788-800.

5. Ruiz Montanez G, Burgos-Hernández A, Calderón-Santoyo M, López-Saiz C, Velázquez-Contreras C, (2015) Screening antimutagenic and antiproliferative properties of extracts isolated from Jackfruit pulp (Artocarpus heterophyllus Lam). Food Chemistry 175: 409-416.

6. Tamang J, Shin D, Jung S, Chae S (2016) Functional properties of microorganisms in fermented foods. Front Microbiol 7: 578.

7. Sreeramulu G, Zhu Y, Knol W (2000) Kombucha fermentation and its antimicrobial activity. J Agric Food Chem 48(6): 2589-2594.

8. Jayabalan R, Subathradevi P, Marimuthu S, Sathishkumar M, Swaminathan K (2008) Changes in free-radical scavenging ability of Kombucha tea during fermentation. Food Chem 109(1): 227-234.

9. Kapp J, Sumner W (2019) Kombucha: A systematic review of the empirical evidence of human health benefit. Ann Epidemiol 30: 66-70.

10. Leal M, Suárez V, Oros H (2018) A review on health benefits of Kombucha nutritional compounds and metabolites. CyTA-J Food 16 (1): 390-399.

11. Aziz N, Koh S, Abdullah R, Hamid N, Mustaffa R (2018) The phytochemical and antioxidant characteristics of fermented jackfruit (Artocarpus heterophyllus l.) leaves using single and mixed starter culture. J Food Sci and Eng 8: 55-60.

12. Wu X, Ran Z, Kong X, Tang Z, Dong X (2010) Functions of microbiota in monogastric gastrointestinal tract and new practices in animal production. J Food Agric Environ 8(2): 695-702.

13. Penders J, Thijs C, Vink C, Stelma F, Snijders B, et al. (2006) Factors influencing the composition of the intestinal microbiota in early infancy. Pediatrics 118(2): 511-521.

14. Tillisch K, Labus J, Kilpatrick L, Jiang Z, Stains J, et al. (2013) Consumption of fermented milk product with probiotic modulates brain activity. Gastroenterology 144(7): 1394-1401.

15. Plé C, Breton J, Daniel C, Foligné B (2015) Maintaining gut ecosystems for health: Are transitory food bugs stowaways or part of the crew? Int Food Microbiol 213: 139-143.

16. Yeoh N, Burton J, Suppiah P, Reid G, Stebbings S (2013) The role of the microbiome in rheumatic diseases. Curr Rheumatol Rep 15(3): 314

17. Maslowski K, Mackay C (2010) Diet, gut microbiota and immune responses. Nat Immunol 12(1): 5.

18. Kim B, Hong V, Yang J, Hyun H, Im J, et al. (2016) A review of fermented foods with beneficial effects on brain and cognitive function. Prev Nutr Food Sci 21(4): 297-309.

19. Salyers A, West S, Vercellotti J, Wilkins T (1977) Fermentation of mucins and plant polysaccharides by anaerobic bacteria from the human colon. Appl Environ Microbiol 34(5): 529-533.

20. Mortensen P, Clausen M (1996) Short-chain fatty acids in the human colon: relation to gastrointestinal health and disease. Scand J Gastroenterol 31(216): 132-148

21. Claus SP, Swann JR (2013) Nutrimetabonomics: applications for nutritional sciences, with specific reference to gut microbial interactions. Annu Rev Food Sci Technol 4: 381-399.

22. Le Blanc J, Chain F, Martín R, Bermúdez Humarán L, Courau S, et al. (2017) Beneficial effects on host energy metabolism of short-chain fatty acids and vitamins produced by commensal and probiotic bacteria. Microb Cell Fact 16(1): 79.

23. Canfora E, Jocken J, Blaak E (2015) Short-chain fatty acids in control of body weight and insulin sensitivity. Nat Rev Endocrinol 11(10): 577.

24. Chambers ES, Preston T, Frost G, Morrison DJ (2018) Role of gut microbiota-generated short-chain fatty acids in metabolic and cardiovascular health. Curr Nutr Rep 7(4): 198-206.
25. Gomes S, Oliveira C, Azevedo Silva J, Casanova M, Barreto J, et al. (2018) The role of diet-related short-chain fatty acids in colorectal cancer metabolism and survival: prevention and therapeutic implications. Curr Med Chem 27(24): 4087-4108.

26. Ulven T (2012) Short-chain free fatty acid receptors FFA2/GPR43 and FFA3/GPR41 as new potential therapeutic targets. Front Endocrinol 3: 111.

27. Eckburg PB, Bik EM, Bernstein CN, Purdom E, Dethlefsen L, et al. (2005) Diversity of the human intestinal microbial flora. science 308(5728): 1635-1638.

28. Chakravorty S, Helb D, Burday M, Connell N, Alland D (2007) A detailed analysis of $16 \mathrm{~S}$ ribosomal RNA gene segments for the diagnosis of pathogenic bacteria. J Microbiol Meth 69(2): 330-339.

29. Wang Y, Qian P (2009) Conservative fragments in bacterial 16S rRNA genes and primer design for $16 \mathrm{~S}$ ribosomal DNA amplicons in metagenomic studies. PloS one 4(10): e7401.

30. Magoč T, Salzberg S (2011) FLASH: fast length adjustment of short reads to improve genome assemblies. Bioinformatics 27(21): 2957-2963.

31. Caporaso JG, Kuczynski J, Stombaugh J, Bittinger K, Bushman FD, et al (2010) QIIME allows analysis of high-throughput community sequencing data. Nature Methods 7(5): 335-336.

32. Haas BJ, Gevers D, Earl AM, Feldgarden M, Ward DV, et al. (2011) Chimeric 16S rRNA sequence formation and detection in Sanger and 454-pyrosequenced PCR amplicons. Genome research 21(3): 494-504.

33. Edgar R (2013) UPARSE: highly accurate OTU sequences from microbial amplicon reads. Nature Methods 10(10): 996-998.

34. De Santis TZ, Hugenholtz P, Larsen N, Rojas M, Brodie EL, et al. (2006) Greengenes, a chimera-checked 16S rRNA gene database and workbench compatible with ARB. Appl Environ Microbiol 72(7): 5069-5072.

35. Lan Y, Wang Q, Cole JR, Rosen GL (2012) Using the RDP classifier to predict taxonomic novelty and reduce the search space for finding novel organisms. PLoS one 7(3): e32491.

36. Caporaso JG, Bittinger K, Bushman FD, De Santis TZ, Andersen GL, et al. (2009) PyNAST: a flexible tool for aligning sequences to a template alignment. Bioinformatics 26(2): 266-267.

37. Bardou P, Mariette J, Escudié F, Djemiel C, Klopp C (2014) jvenn: an interactive Venn diagram viewer. BMC bioinformatics 15(1): 293.

38. Schloss PD, Westcott SL, Ryabin T, Hall JR, Hartmann M, et al. (2009) Introducing mothur: open-source, platform-independent, community-supported software for describing and comparing microbial communities. Appl Environ Microbiol 75(23): 7537-7541.

39. Howe E, Holton K, Nair S, Schlauch D, Sinha R, et al. Mev: multiexperiment viewer. Biomedical informatics for cancer research: Springer $267-$ 277.

40. Bellikci Koyu E, Sarer Yurekli BP, Akyon Y, Aydin Kose F, Karagozlu C, et al. (2019) Effects of regular kefir consumption on gut microbiota in patients with metabolic syndrome: a parallel-group, randomized, controlled study. Nutrients 11(9): 2089.

41. Cotter PD, Bourrie B, Willing BP (2016) The microbiota and health promoting characteristics of the fermented beverage kefir. 7: 647 .

42. Chen D, Yang Z, Chen X, Huang Y, Yin B, et al. (2014) The effect of Lactobacillus rhamnosus hsryfm 1301 on the intestinal microbiota of a hyperlipidemic rat model. BMC Complement Altern Med 14(1): 386.

43. Shin J, Lee S, Go M J, Lee S, Kim S, et al. (2016) Analysis of the mouse gut microbiome using full-length $16 \mathrm{~S}$ rRNA amplicon sequencing. Sci Rep 6: 29681

44. Koliada A, Syzenko G, Moseiko V, Budovska L, Puchkov K, et al. (2017) Association between body mass index and Firmicutes/Bacteroidetes ratio in an adult Ukrainian population. BMC Microbiol 17(1): 120.

45. Ahmed F, Kerna N, Tulp 0 (2019) Managing the F:B Ratio in DM: a re- 
view of the role of Firmicutes and Bacteroidetes in diabetes mellitus Adv Complement Alt Med 4 (1).

46. Hakkak R, Korourian S, Foley SL, Erickson BD (2017) Assessment of gut microbiota populations in lean and obese Zucker rats. PloS one 12(7).

47. Welte C, Deppenmeier U (2014) Bioenergetics and anaerobic respiratory chains of aceticlastic methanogens. Biochim Biophys Acta Bioenerg 1837(7): 1130-1147.

48. Million M, Maraninchi M, Henry M, Armougom F, Richet H, et al. (2012) Obesity-associated gut microbiota is enriched in Lactobacillus reuteri and depleted in Bifidobacterium animalis and Methanobrevibacter smithii. Int J Obesity 36(6): 817.

49. Chaudhary PP, Conway PL, Schlundt J (2018) Methanogens in humans: potentially beneficial or harmful for health. Appl Microbiol Biotechnol 102(7): 3095-3104.

50. Hippe B, Zwielehner J, Liszt K, Lassl C, Unger F, et al. (2011) Quantification of butyryl CoA: acetate CoA-transferase genes reveals different butyrate production capacity in individuals according to diet and age. FEMS microbiology letters 316(2): 130-135

51. Canani R, Di Costanzo M, Leone L, Pedata M, Meli R, et al. (2011) Potential beneficial effects of butyrate in intestinal and extraintestinal diseases. World J Gastroenterol 17(12): 1519.

ISSN: 2574-1241

DOI: $10.26717 /$ BJSTR.2020.29.004817

Soo Peng Koh. Biomed J Sci \& Tech Res

(C) This work is licensed under Creative Commons Attribution 4.0 License

Submission Link: https://biomedres.us/submit-manuscript.php
52. Bourassa M, Alim I, Bultman S, Ratan R (2016) Butyrate, neuroepigenetics and the gut microbiome: can a high fiber diet improve brain health? Neurosci Lett 625: 56-63.

53. Patel B (2018) Sodium butyrate controls cardiac hypertrophy in experimental models of rats. Cardiovasc Toxicol 18(1): 1-8.

54. Wu X, Wu Y, He L, Wu L, Wang X, et al. (2018) Effects of the intestinal microbial metabolite butyrate on the development of colorectal cancer. J Cancer 9(14): 2510.

55. Gonçalves P, Gregório I, Martel F (2011) The short-chain fatty acid butyrate is a substrate of breast cancer resistance protein (BCRP). Am J Physiol Cell Physiol 301(5): C984-C994.

56. Roediger W (1982) Utilization of nutrients by isolated epithelial cells of the rat colon. Gastroenterology 83(2): 424-429.

57. Scheppach W (1994) Effects of short chain fatty acids on gut morphology and function. Gut 35(1): S35-S38.

58. Gao Z, Yin J, Zhang J, Ward R, Martin R, et al. (2009) Butyrate improves insulin sensitivity and increases energy expenditure in mice. Diabetes 58(7): 1509-1517.

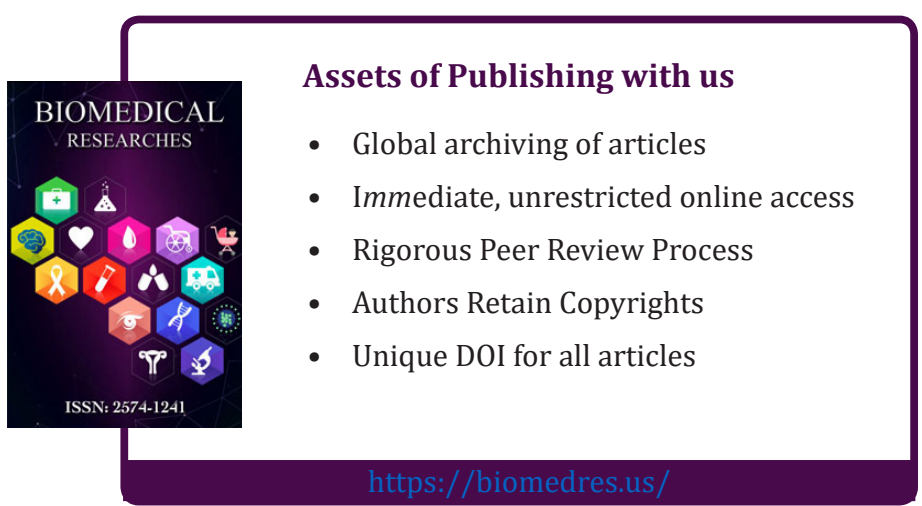

